\title{
Underwater image enhancement algorithm based on dark channel prior and underwater imaging model
}

\author{
Zhengping Sun, Fubing $\mathrm{Li}^{*}$, and Yuying Yang
}

School of Information and Communication Engineering, Beijing Information Science and Technology University, Beijing 100101, China

\begin{abstract}
The main reason for the degradation of the underwater image is the light absorption and scattering. The images are captured in the underwater environment often have some problems such as loss of image information, low contrast, and color distortion. In order to solve the above problems, this paper proposes an image enhancement method for the underwater environment. With the help of the underwater imaging model and dark channel prior theory, a new idea of adding transmission correction and color compensation to $G$ and $B$ color channels is proposed. Experimental results show that, compared with the traditional methods, this method has a better effect on the underwater image with less color deviation.
\end{abstract}

\section{Introduction}

In recent years, the application of underwater environmental monitoring and information collection is more and more extensive, so the collection and research of underwater information with clear underwater images are the most important. However, the particle molecules in the water will cause the scattering of light, and the absorption characteristics of water to light will also cause light attenuation, which will cause poor image quality, blue-green fog surface feeling, low contrast, limiting the visibility, and other defects.

Underwater image quality enhancement can be divided into image restoration methods [1-3] and image enhancement methods [4-6]. The process of image restoration to enhance image quality mainly depends on a specific degradation process and a real imaging model. The process of using the image enhancement method to enhance image quality only needs to pay attention to the pixel value of the image and adjust the pixel value to optimize the overall image quality.

\section{Imaging model of light in the underwater environment}

In the special underwater environment, the attenuation is caused by the absorption and

\footnotetext{
* Corresponding author: lifubing@,bistu.edu.cn
} 
scattering of light by water. Absorption means that part of the light wave will be absorbed by water and converted into heat energy of water in underwater transmission, which causes energy loss. Moreover, the longer the wavelength is, the more obvious the absorption effect is. The scattering is caused by the suspended particles in the water, and the current propagation direction will be shifted.

According to reference [1], the light components arriving at the imaging interface are direct component, forward scattering component, and backscattering component. The direct component only considers the attenuation of the reflected light due to the medium, the reflected light of the object in the underwater scene represents the real information of the object; the forward scattering component is the component with a small-angle deviation of the direct component; the backscattering component is different from the first two components, which is caused by the suspended particles in the underwater environment, and light passing through these particles will scatter at a large angle. Fig. 1 illustrates the imaging model of the underwater environment.

Considering that the forward scattering component has little effect on the phase ambiguity caused by imaging, even if forward scattering accounts for $90 \%$ of the total scattering events, it can also be ignored. Therefore, the imaging model can be simplified as the sum of direct component and backscattering component.

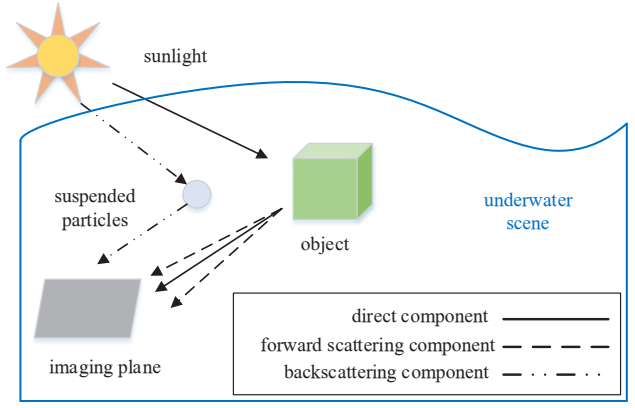

Fig. 1. Underwater optical imaging model.

\section{Dark channel prior algorithm}

Because the underwater imaging model is similar to the fog imaging model, and the contrast and clarity of the image will be reduced due to the attenuation of the medium. Therefore, the dark channel prior to the foggy environment can be applied to the underwater environment.

However, the underwater complex environment is different from the fog environment, because the attenuation of light in different wavebands is variant, so the attenuation is the most serious for the red light with the longest wavelength. If the prior theory of fog image is used in the underwater environment, the value of dark channel will be very low and cannot display any information of the underwater scene. Reference [3] proposed a prior theory of dark channel for the underwater scene, namely Red Channel prior theory. To conform to the prior theory of dark channel, it is necessary to take the red channel inversely, and then split the imaging model into three color channels:

$$
\begin{gathered}
1-I_{R}=t_{R}\left(1-J_{R}\right)+\left(1-t_{R}\right)\left(1-B_{R, \infty}\right) \\
I_{G}=t_{G} \cdot J_{G}+\left(1-t_{G}\right) B_{G, \infty} \\
I_{B}=t_{B} \cdot J_{B}+\left(1-t_{B}\right) B_{B, \infty}
\end{gathered}
$$


The Red Channel prior is as follows:

$$
J^{\operatorname{Red}}=\min \left[\min _{\Omega}\left(1-J_{R}\right), \min _{\Omega} J_{G}, \min _{\Omega} J_{B}\right] \rightarrow 0
$$

Combined with the prior theory of Red Channel, the transmission can be expressed as:

$$
t_{\lambda}=1-\min \left[\frac{\min _{\Omega}\left(1-I_{R}\right)}{1-B_{R, \infty}}, \frac{\min _{\Omega} I_{G}}{B_{G, \infty}}, \frac{\min _{\Omega} I_{B}}{B_{B, \infty}}\right]
$$

Underwater background light $B_{\lambda, \infty}$ in the underwater environment generally takes the pixel value with the highest intensity in the image:

$$
B_{\lambda, \infty}=\max _{I}\left(\max _{\Omega} I_{\lambda}\right)
$$

Combined with the above transmittance and underwater background light, the enhanced underwater image can be expressed as:

$$
J_{\lambda}=\frac{I_{\lambda}-B_{\lambda, \infty}}{t_{\lambda}}+B_{\lambda, \infty}
$$

\section{The improved image enhancement algorithm}

Even considering the different attenuation degrees, wavelength, and transmission of $R, G$, and $B$ channels in the underwater environment, the recovered image is still unsatisfactory. To solve the problems of low contrast and loss of details, the transmission correction and color compensation are proposed in this paper:

1) In the step of Red Channel prior processing image, a correction factor is added in the process of transmission estimation.

$$
t_{\lambda}=1-\min \left[\frac{\min _{\Omega}\left(1-I_{R}\right)}{1-B_{R, \infty}}, \varepsilon_{1} \cdot \frac{\min _{\Omega} I_{G}}{B_{G, \infty}}, \varepsilon_{2} \cdot \frac{\min _{\Omega} I_{B}}{B_{B, \infty}}\right]
$$

2) The idea of shielding $G$ to compensate $R$ and $B$ is adopted, that is adding a part of $G$ to $R$ and $B$. The purpose of color compensation can be achieved by color compensation of image $J$.

$$
\begin{aligned}
& J_{R C}=J_{R}+\beta_{1} \cdot\left(J_{\text {mean }}^{G}-J_{\text {mean }}^{R}\right) \cdot J_{G} \\
& J_{B C}=J_{B}+\beta_{2} \cdot\left(J_{\text {mean }}^{G}-J_{\text {mean }}^{B}\right) \cdot J_{B}
\end{aligned}
$$

$J_{R C}$ and $J_{B C}$ are the $R$ and $B$ components of the final output image.

\section{Experimental results and analysis}

To evaluate the result of the algorithm in this paper, two images with serious color deviation are selected for experimental comparison. Four methods are compared with the original image and the algorithm in this paper, as shown in Fig. 2. Among them, method 1 uses the dark channel prior method; method 2 uses single scale Retinex algorithm; method 
3 uses multi-scale Retinex algorithm; method 4 uses multi-scale Retinex algorithm with color restoration.

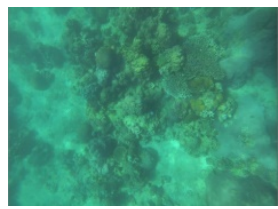

Original image 1

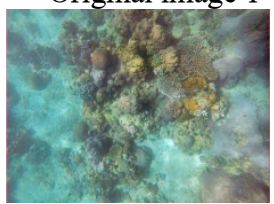

Method 2

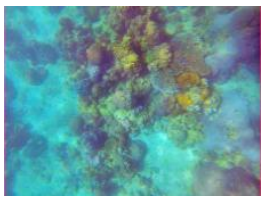

Method 4

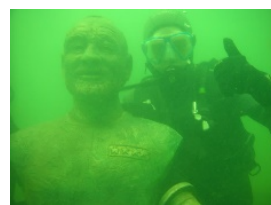

Original image 2
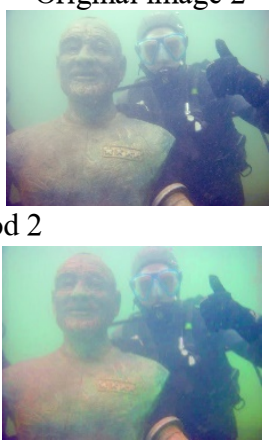

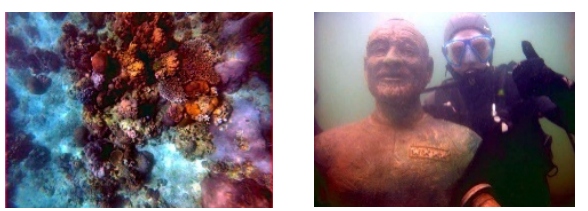

Method 1

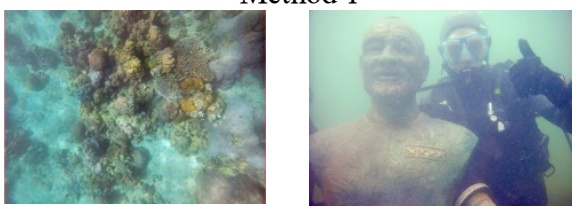

Method 3

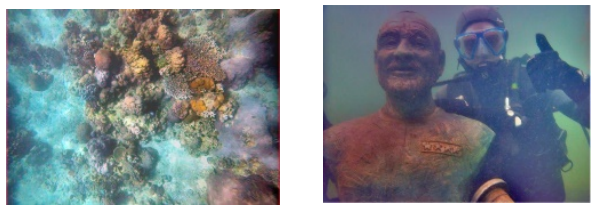

Proposed algorithm

Fig. 2. Comparison of underwater images processed with different methods.

Compared with other methods, the image contrast of the proposed algorithm has been significantly improved, and there is no distortion and over-saturation.

As shown in Table 1, it is an objective index to evaluate image quality. The average gradient and information entropy are used to evaluate the effectiveness of the proposed algorithm and other methods for underwater image restoration. The average gradient represents the mean value of all points in the gradient map corresponding to the image. If the average gradient is large, it means that the image level is rich and varied, and the image quality is better. Information entropy represents the amount of information contained in the image. If the information entropy of the image is larger, the image contains more information and the image quality is higher.

Table 1. Image quality assessment standard.

\begin{tabular}{|c|c|c|}
\hline \multirow{2}{*}{ Method Image 1 } & \multicolumn{2}{|c|}{ Image quality assessment standard } \\
\cline { 2 - 3 } & Average gradient & Information entropy \\
\hline Original image & 2.64 & 13.42 \\
\hline Method1 & 6.45 & 15.81 \\
\hline Method2 & 5.81 & 15.85 \\
\hline Method3 & 5.81 & 15.85 \\
\hline Method4 & 4.30 & 16.43 \\
\hline Proposed algorithm & 7.87 & 16.57 \\
\hline Image 2 & \multicolumn{2}{|c|}{ Image quality assessment standard } \\
\cline { 2 - 3 } Method & Average gradient & Information entropy \\
\hline Original image & 2.64 & 13.42 \\
\hline Method1 & 6.45 & 15.81 \\
\hline Method2 & 5.81 & 15.85 \\
\hline Method3 & 5.81 & 16.85 \\
\hline Method4 & 4.30 & 16.57 \\
\hline Proposed algorithm & 7.87 & \\
\hline
\end{tabular}


It can be judged from the table that the average gradient and information entropy of the proposed algorithm is the highest among the six methods, which means that the image quality processed by the proposed algorithm is the highest.

\section{Conclusion}

Based on the underwater imaging model and dark channel prior theory, combined with transmission correction and color compensation, the underwater image quality is effectively improved. In contrast, saturation and clarity are better than other methods.

This work is supported by Science and Technology Projects of Beijing Municipal Education Commission (KM201911232012), and Qin Xin Talents Cultivation Program, Beijing Information Science \& Technology University (QXTCP C201905).

\section{References}

1. J.S. Jaffe. Underwater optical imaging: the past, the present, and the prospects. IEEE Journal of Oceanic Engineering, 40, 3 (2014) 683-700.

2. H. Wen, Y. Huang and W. Gao. Single underwater image enhancement with a new optical model. IEEE International Symposium on Circuits and Systems, 1 (2013) 753-756.

3. A. Galdran, D. Pardo, A. Picon, et al. Automatic Red-Channel underwater image restoration. Journal of Visual Communication\&Image Representation, 26, 1 (2015) 132-145.

4. D. J. Jobson, Z. Rahman and G. A. Woodell. A multiscale retinex for bridging the gap between color images and the human observation of scenes. IEEE Transactions on Image Processing, 6, 7 (1997) 965-976.

5. D. J. Jobson, Z. Rahman and G. A. Woodell. Properties and performance of a center/surround retinex. IEEE Transactions on Image Processing, 6, 3 (1997) 451-462.

6. J. Wang and H. Bi. Retinex-based Color Correction for Displaying High Dynamic Range Images. Ineternational Confernece on Signal Processing Proceedings. (2010) 1021-1024.

7. K. He, J. Sun, and X. Tang. Single image haze removal using dark channel prior. IEEE Transactions on Pattern Analysis and Machine Intelligence, 33, 12 (2011) 2341-2353.

8. C. O. Ancuti, C. Ancuti, C. De Vleeschouwer and P. Bekaert. Color Balance and Fusion for Underwater Image Enhancement. IEEE Transactions on Image Processing, 27, 1 (2018) 379-393. 